\title{
Algorithm to Analyze the Probability of False Initiation in Order to Provide Seamless Mobility in Next Generation Wireless System
}

\author{
Santosh S. Sambare \\ Department of Computer Engg, Pimpri Chinchwad \\ College of Engineering, Nigdi, Pune
}

\author{
M. U. Kharat \\ Department of Computer Engineering, \\ MET, Nashik
}

\begin{abstract}
Based on the developing trends of mobile communication, next generation wireless networks will have broader bandwidth, higher data rate, and smoother and quicker handoff and will focus on ensuring seamless service across a multitude of wireless systems and networks. The main objective of next generation wireless networks is the integration of independently developed communication networks/systems such as ISDN, Wired/Wireless internetworks and Optical Networks. Proper integration of these emerging technologies will pave a way for convergence of communication systems, information technologies and Internet world with high degree of quality of service along with the global seamless mobility. This paper describes the analysis of false handoff initiation in order to provide seamless mobility in wireless networks.
\end{abstract}

\section{Keywords}

NGWS, False Handoff Initiation, Seamless Handoff, GPS, cellular structure.

\section{INTRODUCTION}

Next Generation Wireless System (NGWS) should satisfy the requirement of seamless mobility as surveyed in [2] without loss and delay of packets and disruption to data communications, increasing the data throughput rate, increasing system capacity (spectral efficiency) without increasing the SNR, decreasing power emitted by the antenna and respectively decreasing the adverse effect of the power emitted on human's health, high accuracy (low BER) data communications even in existing interference (collision),separating useful signal from interference and distributing these signals to different channels and simultaneously receiving and processing both useful and interference signals, separating the direct signal from the reflected signals in the multipath case and distributing these signals to different channels and simultaneously processing both direct and reflected signals. Seamless handoff provides end-to-end IP continuity without failures in the midst of a download activity due to link outages or handovers. What this means is that a user or application that is connected to a handoff server could seamlessly and transparently switch back and forth between different internet technologies, without compromising any communication activity. Seamless handoff ensures successful data transfer even when the underlying link connections are damaged or disrupted due to device mobility. Furthermore, it guarantees this lossless Quality-of-Service when a mobile user or application passes through different alternative technologies.

\section{FALSE HANDOFFS INITIATION}

Seamless handoff provides end-to-end IP continuity without failures in the midst of a download activity due to link outages or handovers as discussed in [1]. What this means is that a user or application that is connected to a handoff server could seamlessly and transparently switch back and forth between different internet technologies, without compromising any communication activity. Seamless handoff ensures successful data transfer even when the underlying link connections are damaged or disrupted due to device mobility. Furthermore, it guarantees this lossless Quality-of-Service when a mobile user or application passes through different alternative technologies. Handoff is initiated when the (received signal strength) RSS of the current serving (base station) BS drops below a predefined fixed threshold value. While Mobile Terminal (MT) is moving, RSS of the base station A is starting to drop below the threshold value $S_{\text {th }}$ at point $P$. Therefore, MT will be in handoff process soon, If RSS of base station B is larger than or more optimized than RSS of base station A then MT will send handoff registration message to base station $\mathrm{B}$. We assume that MT can move in any direction with equal probability.

As shown in the Figure 1. [5], heterogeneous wireless networks configure with different network architectures such as pico-cell, micro-cell and satellite systems, etc. Mobile Terminal (MT) is currently located in the coverage area of cell A and it may be possibly moving into cell area of B or cell $\mathrm{E}$ or cell $\mathrm{J}$, respectively. The future location of an MT depends on its moving direction. MT's current position is closely related to its next position due to the continuity of movement. If MT is moving to east, it is likely that it is going to move into cell B. Currently MT's direction changes from east to north it is most likely that it is going to move to cell $\mathrm{E}$. Therefore incorporation of mobile terminals (MT) movement direction and position is very important to provide an accurate mobility pattern for handoff.

In current though MT's coming direction had already changed to cell $\mathrm{E}$, its direction can change to cell $\mathrm{J}$ also. Future communication environment has been composed of different types of heterogeneous wireless networks which are overlapped with each other. Therefore, MT currently in base station A can be possible handoff process to most networks. In wireless networks, the movement patterns of mobile objects are very important because mobility and resource management play in vital role for supporting mobility and providing QoS. Let's assume the shape of each cell is hexagon and it has six neighbours. In that case although we can assume the probability of an MT leaving along one side is $1 / 6$, it is false for the MT's with an active connection and a specific destination. 


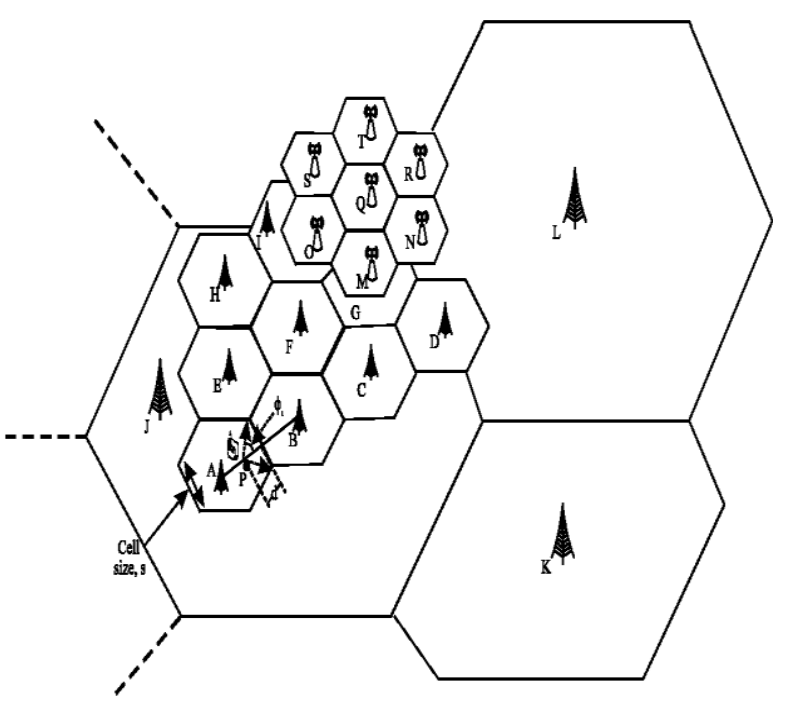

Figure 1: False handoff Initiation scenario

If we can know MT's current precise position and direction, we can decrease the false handoff initiation probability as described in to nearly zero. The more we can predict MT's current position and direction exactly, the more accurate the probability of handoff initiation we get as discussed in [5].

\section{ALGORITHM AND ANALYSIS OF FALSE HANDOFF INITIATION}

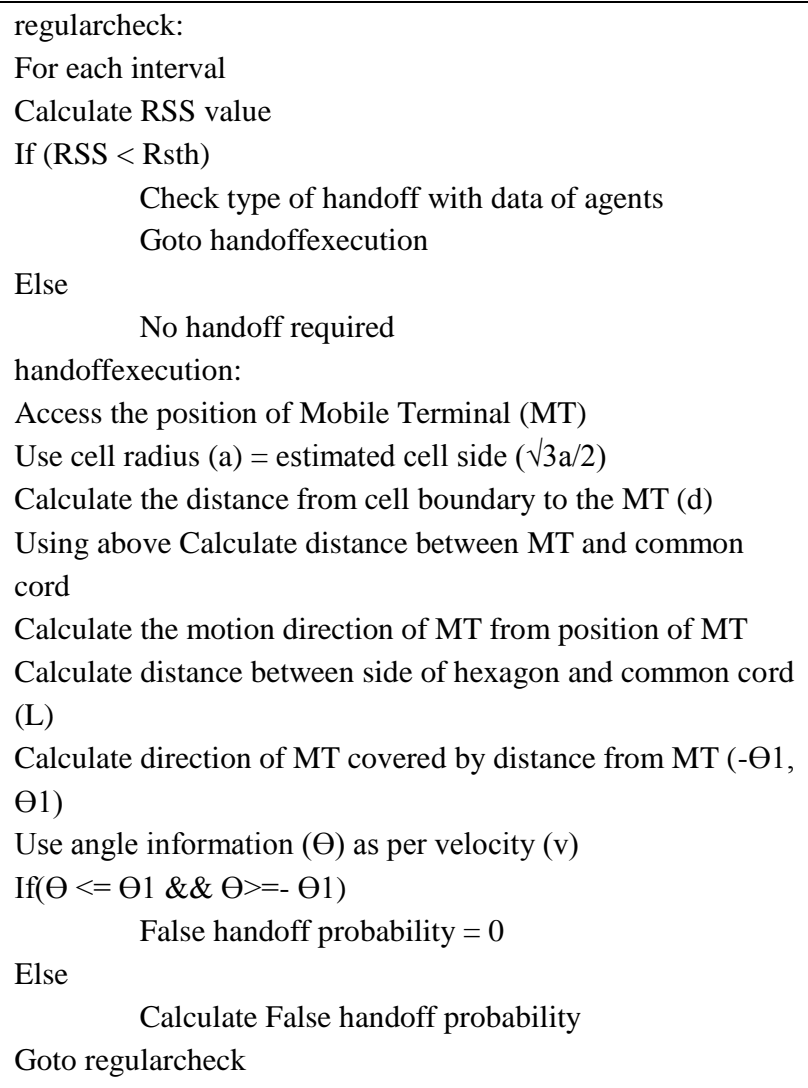

Calculate False handoff probability

Previously, we have discussed why the shape of the cell is considered to be a regular hexagon. We have seen two cells overlapping in such a manner that the common cord between two adjacent circular cells also becomes the common side of the regular hexagons, when the cells are considered to be hexagons. However, two cells may overlap in such a way that there is some overlapping hexagonal portion between them as shown in Figure 2.

Taking the references of [6],[7],[8],[9] and [10] survey and analysis is being done. $\mathrm{AB}$ is the side of regular hexagonal cell served by the Old BS (OBS). But $A^{\prime} B^{\prime}$ is the common cord of the two adjoining cells, one served by the OBS and the other by the NBS. When a Mobile Terminal (MT) crosses $A^{\prime} B$ ', then it will be under the New BS (NBS). This is because RSS of NBS is greater then RSS of OBS to the right side of $A^{\prime} B$ '. Once the MT reaches the boundary of the circular cell (real) then the MT discovers that it may enter into the coverage area.

$$
\begin{aligned}
& \mathrm{QR}=\mathrm{L} \\
& \mathrm{PQ}=\mathrm{d}
\end{aligned}
$$

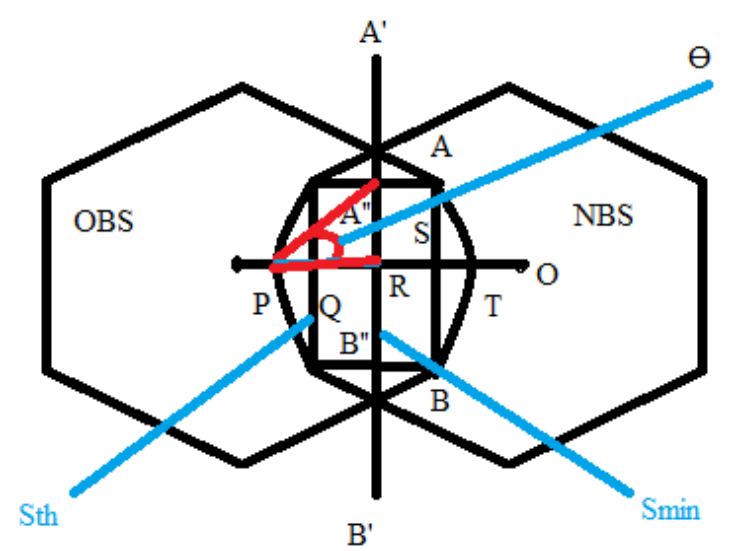

Figure 2: Handoff Analysis for Partially overlapped cells

Here, we have considered some hexagonal portion to be overlapping. MT is moving from its current serving BS (old $\mathrm{BS}$ ), to the future serving BS (new BS) from which the probability of false handoff is calculated.

The following are the definitions of the notations used in the figure:

Sth : The RSS threshold value to initiate the handoff. This implies, when RSS of OBS goes below Sth, the Hierarchical Mobile IP (HMIP) registration procedures are initiated for MT's handover to the NBS.

Smin: The MT's minimum RSS value to communicate successfully between an MT and BS.

OBS: The old BS.

NBS: The new BS.

Here a: The cell size served by a BS (i.e., the length of each side of hexagonal cells).

P: The point when the MT's RSS from the OBS drops below Sth. On the point 'P' the MT understands that it is on the overlapping position.

Here d: The distance from the hexagonal cell boundary to the point 'P'.

Here $\Theta$ : The motion direction of MT from point ' $\mathrm{P}$ ' to handoff to NBS.

L: Distance between side of hexagon and common cord of two hexagons. 
$\beta$ : Any direction of MT when at time ' $t$ ' it covered the distance ' $\mathrm{x}$ ' from the point ' $\mathrm{P}$ '.

$\mathrm{AB}=\mathrm{a}=$ radius of the circle $=$ length of the side of proposed hexagon.

$\mathrm{OQ}=\sqrt{3} \mathrm{a} / 2$

$\mathrm{PQ}=\mathrm{d}=\mathrm{OP}-\mathrm{OQ}$

$=\mathrm{a}-(\sqrt{ } \mathrm{3} \mathrm{a} / 2)$

$=(2 a-\sqrt{3} a) / 2$

$\mathrm{QR}=\mathrm{L}$ (assumption)

When MT crosses the line $A^{\prime} B^{\prime}$, only then handoff will occur.

$\mathrm{PR}=\mathrm{PQ}+\mathrm{QR}$

$=\mathrm{d}+\mathrm{L}$

$=(2 \mathrm{a}-\sqrt{3} \mathrm{a}+2 \mathrm{~L}) / 2$

$\mathrm{A}^{\prime} \mathrm{A}^{\prime \prime}=\mathrm{L} \tan 30^{\circ}$

$=\mathrm{L} / \sqrt{3}$

$A^{\prime} R=(\sqrt{ } 3 a+2 L) / 2 \sqrt{ } 3$

$X=((2 a-\sqrt{3} a+2 L) \sec \theta) / 2$

$\mathrm{t}=((2 \mathrm{a}-\sqrt{3} \mathrm{a}+2 \mathrm{~L}) \sec \theta) / 2 \mathrm{v}$

Where ' $v$ ' is the velocity of MT.

$\tan \theta=\mathrm{A}^{\prime} \mathrm{R} / \mathrm{PR}$

$=(\sqrt{3} \mathrm{a}+2 \mathrm{~L}) / \sqrt{ } 3(2 \mathrm{a}-\sqrt{3} \mathrm{a}+2 \mathrm{~L})$

A scenario where an MT is currently served by OBS is considered for the analysis. We consider that the MT is moving with a speed ' $\mathrm{V}$ '. ' $\mathrm{V}$ ' is assumed to be uniformly distributed in [Vmin, Vmax]. So we can say that the probability density function (pdf) of ' $\mathrm{V}$ ' is given by:

$\mathrm{F}(\mathrm{v})=1 / \mathrm{V} \max -\mathrm{Vmin}$

Where $V \min <\mathrm{v}<\mathrm{Vmax}$

During the course of movement the MT discovers that it is going to move into the subnet served by NBS. It is assumed that during the course of its movement when the MT reaches the point $\mathrm{P}$, the RSS from OBS goes below Sth. So, when MT reaches P, the HMIP [3] registration is initiated with the New FA (NFA). At this point, the RSS received by the MT from NBS may not be sufficient for the MT to send the HMIP registration messages to NFA through NBS. Hence, the MT may send the HMIP registration message to NFA through OBS. This is called pre-registration. For a smooth and successful handoff from OBS to NBS, MT's HMIP registration with NFA and link layer associations with NBS must be completed before the RSS of OBS goes below Smin, i.e., before the MT moves beyond the coverage area of OBS. When the MT is located at point $\mathrm{P}$, it is assumed that it can move in any direction with equal probability as in [4] i.e., the probability density function of MT's direction $\Theta$ is:

$\mathrm{F}(\phi)=1 / 2 \Pi$

where $-\Pi<\phi<\Pi$

$\phi \in\left(-\phi_{1}, \phi_{1}\right)$.

The probability of false handoff initiation can be calculated as follows:-

$\mathrm{P}=1-\int_{-\phi 1}^{\phi 1} f(\phi) \mathrm{d} \phi$

Now if $\int_{-\phi 1}^{\phi 1} f(\phi) \mathrm{d} \phi=0$ then the Mobile Terminal is not between $-\phi_{1}$ and $\phi 1$

This states that there is sudden change in the direction of mobile terminal. Hence it is clear that in such cases the probability of false handoff will be 100 percent

i.e. $\mathrm{P}=1-0=1$

where $\Theta=\arctan ((\sqrt{3} \mathrm{a}+2 \mathrm{~L}) / \sqrt{ } 3(2 \mathrm{a}-\sqrt{3} \mathrm{a}+2 \mathrm{~L}))$

\section{SIMULATION RESULTS AND} ANALYSIS

Following input parameters are needed:

1) Distance between the Cell side and intersection of two cells (L)

2) Cell size (A)

3) Velocity of MT in $x$ direction $(V x)$

4) Velocity of MT in y direction (Vy)

Using the above input parameters False handoff Initiation probability is calculated in two representations:

1) Partially overlapped with respect to $L$ and $A$

2) Partially overlapped with respect to velocity

Analysis with respect to $\mathrm{L}$ and $\mathrm{A}$ is given below:

In the Figure 3, the length (L) value describe the overlapping of two cells, the more the value of $\mathrm{L}$ the more the complex overlapping and as the $\mathrm{L}$ value increases probability of false handoff initiation increases. As decision making for handoff proceeding is necessary in order to avoid false handoff initiation it is better if the cell size (A) is large. So as the cell size (A) decreases and $\mathrm{L}$ value increases, the probability of false handoff initiation increases.

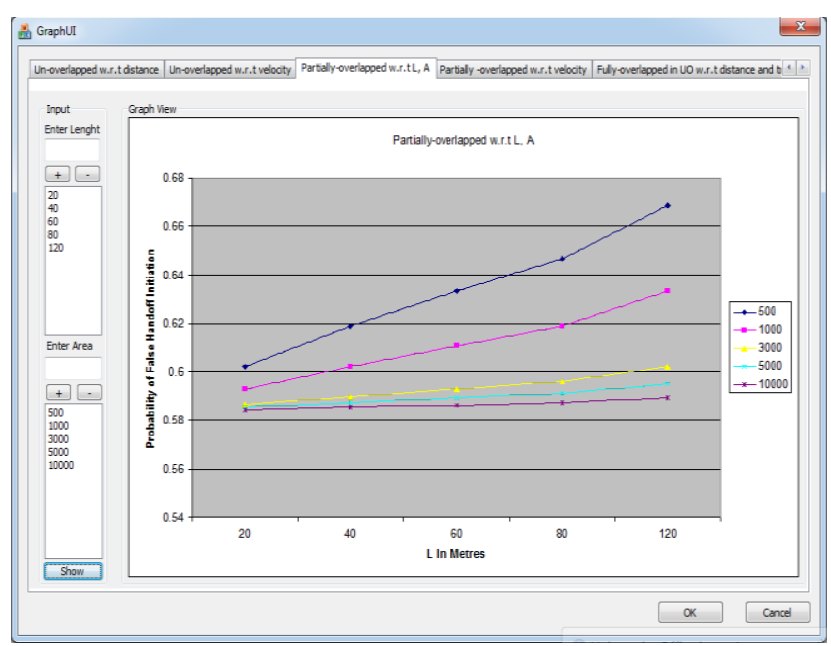

Figure 3. Relationship between $L$ and probability of false handoff initiation with respect to cell size

Analysis with respect to velocity information of MT as shown in Figure 4 and Figure 5.:

Here we are assuming the velocity of MT in $\mathrm{x}$ and $\mathrm{y}$ direction maximum to be $100 \mathrm{~m} / \mathrm{sec}$ but it can be increased in order to check for its subsequent result. Using this velocity the angle is calculated. This angle is supposed to be in between the two $\Theta 1$ and $-\Theta 1$ which state that MT with such velocity for particular cell size will have zero probability of false handoff initiation but if the MT velocity angle is outside the range of $\Theta 1$ and $-\Theta 1$ then probability of false handoff initiation is hundred percent.

In the Figure 4, values of $-\Theta 1$ and $\Theta 1$ is calculated for each of the $\mathrm{L}$ value for different cell size (A). We can see that value of $\Theta 1$ increases as the $\mathrm{L}$ decreases and cell size increases.

So the more the cell size, the more angular part can be traversed by the MT to avoid false handoff initiation. In the graph as shown in Figure 5, we have inputted the value of velocity of MT in $\mathrm{x}$ and $\mathrm{y}$ direction 
$(100,10)$

$(100,20)$,

$(10,100)$,

$(20,100)$ respectively.

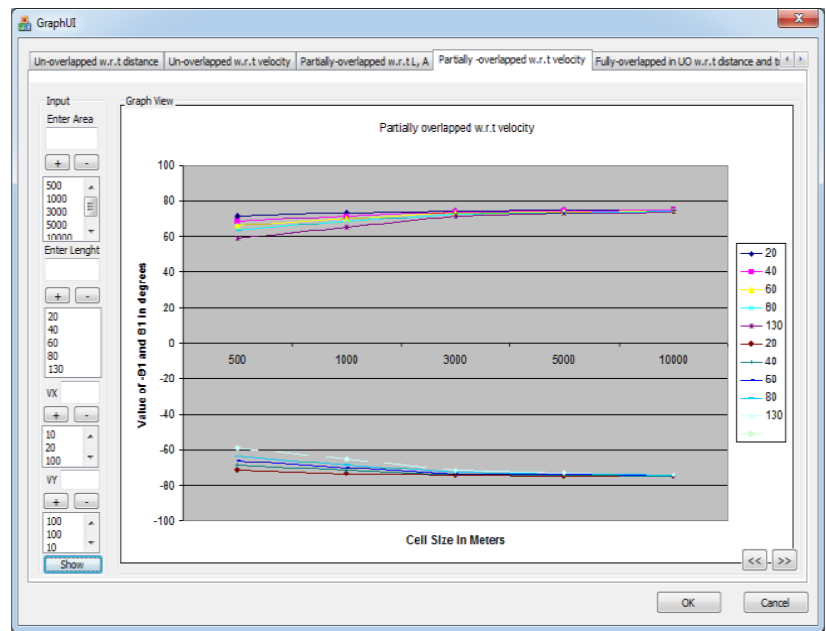

Figure 4. Relationship between cell size and values of - $\theta 1$ and $\Theta 1$ with respect to $L$

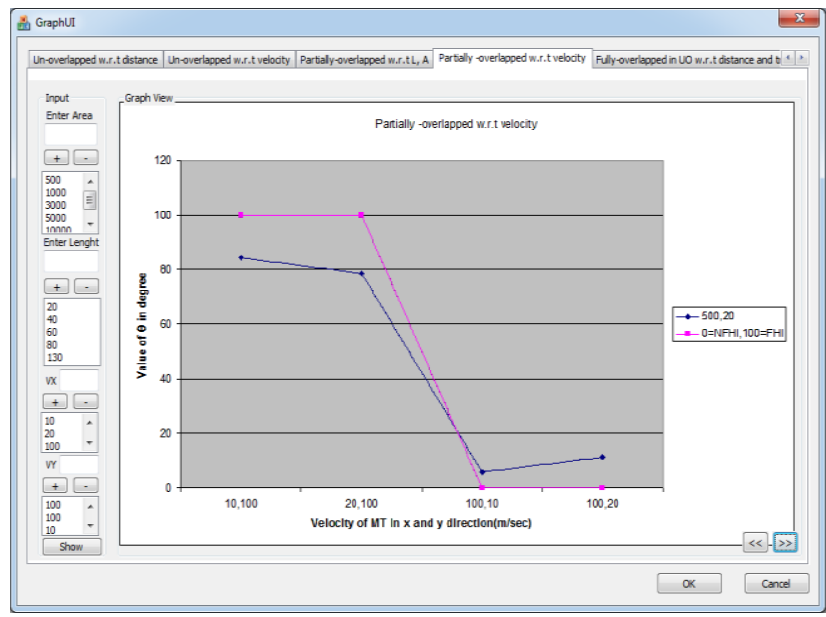

Figure 5. Relationship between velocity of MT and value of $\Theta$ with respect to Cell size and $L$

Using this velocity information the angle $\Theta$ is calculated:

The blue line represent the angel $\Theta$ for each of the velocity of MT for each of the L value for different cell size (A).

The pink line in the graph denotes 2 things:

1) 0 value (for $\Theta$ value in between $\Theta 1$ and $\Theta 2$ ) It states No False handoff Initiation $(\mathrm{NFHI})=$ true

2) 100 value (for $\Theta$ value not in between $\Theta 1$ and $\Theta 2$ ) It states False handoff Initiation $(\mathrm{FHI})=$ true

\section{CONCLUSION}

Handoff Failure is the major concern in the next generation heterogeneous wireless system. This document gives a brief analysis of false handoff initiation and the algorithm to detect such cases of handoff initiation to minimize the probability of handoff failure in heterogeneous wireless system. The algorithm presented in this report illustrate the need to identify the type of handoff and further execution of handoff in order to pre identify the false handoff initiation at early instances and prevent the system from handoff failure which in turn can lead to low packet loss and low handoff signalling delay and provide seamless handoff. In this paper we have described the cellular system along with the need for seamless handoff in order to provide seamless characteristics such as reduction in handoff failure, packet loss, handoff signalling delay with Quality of service. We have mentioned the process of handoff analysis for cells where we have calculated the probability of false handoff initiation taking into consideration the Mobile terminal position and value of $\mathrm{L}$ at each interval in order to prevent from handoff failure by pre identifying the probability of false handoff initiation.

\section{REFERENCES}

[1] Robert Hsieh, Zhe Guang Zhou, Aruna Seneviratne ed." S-MIP: A Seamless Handoff Architecture for Mobile IP" 0-7803-7753-2/03/17.00 (C) 2003 IEEE

[2] Ian F. Akyildiz ed."A Survey of mobility management in next generation All-IP-Based wireless Sys-tem"2004 IEEE

[3] Aisha H. A. Hashim, Farhat Anwar, Shaffah Mohd and Hatina Liyakthalikh ed. "Mobility Issues in Hierarchical Mobile IP" SETIT 2005 3rd International Conference March 27-31, 2005 - TUNISIA

[4] Shantidev Mohanty and Ian F. Akyildiz, Fellow, IEEE" A Cross-Layer (Layer $2+3$ ) Handoff Management Protocol for Next-Generation Wireless Systems" IEEE TRANSACTIONS ON MOBILE COMPUTING, VOL. 5 , NO. 10, OCTOBER 2006

[5] ThaZin Ei and Wang Furong "Cross layer handoff management algorithm on heterogeneous wireless system"IT journal 7(5),820-824,2008.

[6] Koushik Kumar Nundy, Himanish Mukherjee "Minimisation of Hand-off Failure Probability and False Hand-off Initiation in Low Latency Next Generation Wireless Networks", Manuscript received December 5, 2009.

[7] Debabrata Sarddar, Tapas Jana, Souvik Kumar Saha, Joydeep Banerjee , Utpal Biswas, M.K. Naskar "Minimization of Handoff Failure Probability for NextGeneration Wireless Systems". International Journal of Next-Generation Networks (IJNGN) Vol.2, No.2, June 2010

[8] E. Arun, Dr. R.S.Moni "Optimization Algorithm for a handoff decision in wireless heterogeneous networks", International Journal of Next-Generation Networks (IJNGN) Vol.2, No.3, September 2010

[9] Debabrata Sarddar, Shubhajeet Chatterjee, Ramesh Jana, Shaik Sahil Babu, Prabir K Naskar, Utpal Biswas and M.K. Naskar." Minimization of Handoff Failure by Introducing a New Cell Coverage Area in the Handoff Region" IJCSI International Journal of Computer Science Issues, Vol. 8, Issue 3, No. 2, May 2011 ISSN: 1694-0814

[10] Shreya Mallik, Shubhajeet Chatterjee, Pulak Mazumder" Minimization of Call Blocking Probability using Auxiliary Stations" International Journal of Computer Applications (0975 - 8887) Volume 25-No.7, July 2011 